\title{
Is radiotherapy is good or bad?
}

\author{
Shaik Ali Hassan ${ }^{1 *}$, Rinda Sharma ${ }^{2}$, Sumit Bhateja ${ }^{3}$, Geetika Arora ${ }^{4}$ \\ ${ }^{\mathbf{1}, 2}$ Dental Surgeon, ${ }^{3} \mathrm{HOD},{ }^{4}$ Reader, ${ }^{3}$ Dept. of Oral Medicine \& Radiology, ${ }^{4}$ Dept. of Public Health Dentistry, ${ }^{\mathbf{1} 3}$ Manav Rachna Dental \\ College, Faridabad, Haryana, ${ }^{2}$ Amrita Dental College, Kochi, Kerala, ${ }^{4}$ Inderprastha Dental College, Sahibabad, Uttar Pradesh, India
}

\section{*Corresponding Author: Shaik Ali Hassan}

Email: alishaikhassan@gmail.com

\begin{abstract}
Radiation therapy is the treatment of ionizing radiation diseases. Ionizing radiation used for therapeutic purposes. It has high energy radiation in the megavoltage range. Radiation therapy can be used for curative or paliative treatment. Radiation therapy has many applications in nonmalignant conditions like the treatment of trigeminal neuralgia, disease of the thyroid gland, pterygium, pigmented synovitis, prevention keloid scar growth and prevention of heterotopic ossification. In this article we discuss about the role of Radiation therapy and its clinical effects.
\end{abstract}

Keywords: Radiotherapy, Oncologist, Therapeutic, Palliative.

\section{Introduction}

Oncologists first cured from historical record, the first cancer case in 1898. Later on charged particles were allowed to travel to the cancerous cells deep without affecting the skin. Two scientists Stanford, Dr. Henry Kaplan and Edward Ginzton, worked together to take this to a standard where it could be Used in a clinical setting. In 1960, the work was done by these Two researchers have launched the first audience rotatable radiotherapy called linac. Latest advancement in radiotherapy is the Image Guided Radiation Treatment (IGRT). According to the principle states that the rapidly proliferating cells are sensitive to ionizing radiation compared to normal cells, this property is used in therapeutic treatment. ${ }^{1,2}$ It is indicated in diseases like Hodgkin's disease (lymphadenoma) Lymph follicular reticulosis, other reticulosis, chronic myeloid leukaemia, Chronic lymphoid leukaemia. Palliative radiotherapy remains possible for most of the inoperable but not hopelessly advanced cases. ${ }^{3}$ In case of carcinoma of the body of the uterus, vulva, penis, skin of the trunk and limbs, and decision has to be made for each case individually whether to do surgery or radiotherapy. Radiotherapy indicated but of limited value in carcinoma of the larynx, carcinoma of the pharynx, thyroid tumors, malignant salivary gland tumors. Treatment by radiotherapy not good in cases like carcinoma of the stomach, intestine, colon or rectum, hypernephroma, carcinoma of the prostrate, secondary carcinoma in liver or lung, besides limited surgical treatment or hormonal therapy, treatment is of little value. There are generally 2 types of radiotherapy in the-Curative therapy- the intention is to eradicate the disease permanently in the treated area. Palliative therapy- the aim is to achieve temporary improvements in the patient's conditions in circumstances where experience has shown that cure is rarely possible. $^{4}$

External (transcutaneous) irradiation: irradiation of Remote sources of the body (X-rays, tele-therapy) with radius 226, cobalt 60 or cesium 137), Local irradiation (brachytherapy): irradiation of Source in direct contact with the tumor surface irradiation with applicators with radioactive material (mold for the treatment of certain
Tumors such as hard palate and skin carcinomas die). intracavitary irradiation with radioactive material (most often, radius 226, cobalt 60 , cesium 137) in removable applicators inserted in the body Cavities such as the uterus, vagina, nasopharynx or maxilla Sinus. interstitial irradiation with removable needles It contains radio-226, cobalt-60, cesium-137; not removable "seeds" of radioactive gold 198 or radon, by small sources of radioactive iridium in the nylon suture or radioactive tantalum thread 182. Radioisotopes are implanted in the tumor of Carcinoma of the tongue and oral mucosa. Direct radiographic treatment of epithelial lesions by cones. Internal or systemic irradiation: irradiation by radioactive sources administered (32 P, 131 I) intravenously or parenterally Radioactive iodine is used for the treatment of thyroid cancer and Phosphorous-32 is used to treatment of policy themia vera.

\section{Principle of radiotherapy}

Ionizing radiation during passage through the tissue of a patient affects the biology of both normal and tumor tissue. This radiation causes so much direct and indirect effects on biological objectives. Radiation kills by interacting with interfering water molecules that are biochemical processes in the cells. The DNA of a cell can be directly influenced by secondary electrons. The radiation generated as ionizing radiation interacts with the tissue. Radiation can also have an indirect effect due to free radical formation, these radicals in turn can cause most of the chemical damage to DNA. In addition, there are other cellular functions that are disturbed by radiation induced damage of Chromosomal damage also occurs. This damage is modified by oxygen concentration, temperature and others Intercellular components. The fundamental objective of radiotherapy is the destruction of Tumor, but adjacent normal tissue ionizing radiation deposits energy that damages or destroys cells and damage the genetic material, making it impossible for cells, by damaging their genetic material, it is impossible that these cells continue to grow. ${ }^{5}$ 


\section{Complications of radiotherapy}

1. Radiation dysphagia: Most troubleshooting and is least treatable. Patient complains of difficulty in swallowing and food getting stuck in throat. It is caused by radiation fibrosis within the pharyngeal constrictors.

2. Radiation Induced Trismus: Radiation induced trismus without osteoradionecrosis is unique and difficult to treat.

3. Xerostomia due to atrophy of salivary glands.

4. Radiation induced caries which are seen at cervical third of crown.

5. Osteonecrosis of bone.

\section{Managing the radiotherapy treated patients}

Before therapy- Caries control, oral prophylaxis, flouride application. Instruct patient about oral hygiene. Remove teeth with apical pathosis or advanced periodontal disease. Along with any sound teeth in mandible that will be in direct path of radiation. Radiotherapy after 21 days of extraction.

\section{During therapy}

Patient may complain of toothache or dental abscess, no invasive treatment should be given, non invasive methods such as pulpotomies, pulpectomies, endodontic treatment analgesics should be followed. No interruption in radiotherapy is required as the cancer cells may repopulate with more radiation resistant cells. ${ }^{6}$

After therapy-require good oral hygiene, first four months are known as golden window period, necessary oral surgical procedures can be done without HBO therapy. ${ }^{7}$

\section{Conclusion}

Cancer is a life threatening condition as it is detected late so if detected it has to be cured as it has very high metastatic property. With this review article we want to say that there are good and adverse effects of use of radiotherapy. There should be correct knowledge of carcinomas and should know the differential stage treatment like use of therapeutic or palliative treatment.

\section{Source of funding}

None.

\section{Conflict of interest}

None.

\section{References}

1. Fukumoto EK, Barker GJ, Carter-Hanson C, Barker BF. Subjective and clinical evaluation of oral lubricants in xerostomic patients. Spec Care Dent 1998;18:113-8.

2. Ceremonies G, Bryden G, Bottcher C. A multidisciplinary team approach to preservation of quality of life for patients following oral cancer surgery. OHL Head Neck Nurs 2000;18:6-11.

3. Epstein JB, Emerton S, Le ND, Stevenson-Moore P. A doubleblind crossover trial of Oral Balance gel and Biotene toothpaste versus placebo in patients with xerostomia following radiation therapy. Oral Oncol 1999;35:132-7.

4. Makkonen TA, Bostrom P, Vilja P, Joensuu H. Sucralfate mouth washing in the prevention of radiation-induced mucositis: a placebo controlled double-blind randomized study. Int J Radiat Oncol Biol Phys 1994;30:177-82.

5. Nusair S, Rubinow A. The use of oral pilocarpine in xerostomia and Sjogren's syndrome. Semin Arthritis Rheum 1999;28:360-7.

6. Hawthorne M, Sullivan K. Pilocarpine for radiation-induced xerostomia in head and neck cancer. Int J Palliat Nurs 2000;6:228-32.

7. Feber T. Mouth care for patients receiving oral irradiation. Prof Nurse 1995;10(10):666-70.

How to cite this article: Hassan SA, Sharma R, Bhateja $\mathrm{S}$, Arora G. Is radiotherapy is good or bad? Int J Maxillofac Imaging 2019;5(3):56-7. 